\title{
STRATEGY OF THE MUTUAL SEARCH FOR CIVILIZATIONS BY MEANS OF PROBES
}

\author{
U. N. Zakirov \\ Physical-Technical Institute of the USSR Academy of \\ Sciences, 10/7, Sibirski Tract, Kazan 420029, USSR
}

\section{Poster Paper}

The strategy of mutual search for civilizations by means of probes is based on the considerations of the technological level required to utilize internal (rocket-borne) and external (cosmic) energy sources. The possibility of detecting internal energy sources is most developed because well-known physical laws are involved, e.g., the process of energy release from hydrogen isotopes at the expense of microtarget supercompression. It is possible to suggest several tests to search for evidence of internal energy sources in the vicinity of the 50 nearest stars ( 16 light-years). For example, it might be possible to detect power changes in a probe engine from the behavior of its kinematic characteristics as it moves to the nearest stars or from these stars toward the Sun. On the other hand, it might be useful to modify the operating plan for the infrared cameras and Multi-Object Spectrometer of NICMOS-type, which will, for several years, be parts of the Hubble telescope. These instruments could be programmed to search not only for extra solar planets, but for "self-luminous" probes as well.

The possibilities of detecting cosmic energy sources from interstellar probes are speculative. We can only speculate that hypothetical civilizations have developed and are using such technologies as probe accelerations by Ramjets, or the utilization of electric and magnetic fields, or strong gravitational fields, or antigravitation and vacuum energy. 